\title{
Dünnschichtchromatographische Untersuchungen über die Ausscheidung einiger Phenol- und Indolsäuren im Harn
}

\author{
Herrn Prof. Dr. N. Henning zum 70. Geburtstag gewidmet \\ Von E. Scimid, B. Laudi ${ }^{1}$ ), J. Krautheim und N. A. Tautz \\ Aus der Mediziniscben Klinik der Universität Erlangen-Nürnberg (Direktor: Prof. Dr. N. Henning)
}

(Eingegangen am 13. Oktober 1965)

\begin{abstract}
Dünnschichtchromatographische Trennverfahren und Nachweismethoden mit Fluoreszenzlöschung und Färbung werden für 13 Phenolsäuren und 2 Indolsäuren angegeben. Die p-Hydroxyphenylessigsäure läßt sich mit einem neuen Test semiquantitativ nachweisen, der die photochemische Umwandlung der Substanz auf der DC-Platte nach chromatographischer Entwicklung benützt. Orientierende Versuche mit Tyrosin- und Tyramin-Belastung beim Menschen ergeben, daß diese Stoffe zum Teil in Form der „freien“ Phenolsäure mit dem Harn ausgeschieden werden.

Für die Diagnose des Phaeochromozytoms reicht der semiquantitative Nachweis der Vanillinmandelsäure aus. Bei der Diagnostik maligner Sympathikustumoren werden neben erhöhten Vanillinmandelsäuremengen DopaminMetaboliten, wie Homovanillinsäure und Dihydroxyphenylessigsäure sowie die Vanillinșäure vermehrt gefunden. Beim Carcinoid-Syndrom finden sich im Harn große Mengen von 5-Hydroxyindolessigsäure, während die sauren Katecholamin-Metaboliten nicht vermehrt sind. Die Dünnschichtchromatographie mit verschiedenen Fließmittelsystemen eignet sich zum Nachweis dieser Stoffwechselprodukte.

Quantitative Bestimmungen ergeben eine Zunahme der Ausscheidung von Vanillinmandelsäure und 5-Hydroxyindolessigsäure nach oraler Zufuhr von Segontin im akuten Versuch. Bei einwöchiger Einnahme des Pharmakons sinkt die Ausscheidung dieser. Metaboliten wieder annähernd auf den Ausgangswert ab. Die Vanillinsäure kann in Einzelfällen bei längerer Zufuhr von Segontin ansteigen.
\end{abstract}

13 Phenolic and 2 indole acids were separated by thin layer chromatography; fluorescence-quenching and colour development methods are given for their detection. p-Hydroxyphenylacetic acid can be determined semi-quantitatively by a new test, which involves the photochemical conversion of the substance on the thin layer plate after chromatographic development. After loading human subjects with tyrosine or tyramine, only small amounts of these materials were excreted as "free" phenolic acid in the urine.

The semi-quantitative determination of vanilmandelic acid is suitable for the diagnosis of phaeochromocytoma. In the diagnosis of malignant sympathic tumours, there are increased levels of dopamine metabolites, like homovanillic acid and dihydroxyphenylacetic acid, together with vanillic acid, as well as increased amounts of vanilmandelic acid. In the carcinoid syndrome, large amounts of 5-hydroxyindole acetic acid are found in the urine, while the acidic catecholamine metabolites are not increased. Thin layer chromatography with various solvent systems is suitable for the detection of these metabolites.

Quantitative determinations show that there is an increased excretion of vanilmandelic acid and 5-hydroxyindole acetic acid after the oral administration of segontin in acute cases. After taking segontin for one week, the excretion of these metabolites decreases and approaches the starting value. In individual cases and administration of segontin for longer periods, the vanillic acid level may incerase.

Die Untersuchung des Harns auf Phenolsäuren und Metaboliten des Indolstoffwechsels beschränkt sich im allgemeinen auf die Substanzen, die bei Tumoren des sympathiko-adrenalen und entero-chromaffinen Systems, d. h. beim Neuroblastom und Phaeochromozytom sowie beim malignen Carcinoid vermehrt ausgeschieden werden. Für einzelne Stoffe, vor allem die Homovanillinsäure („HVS“), die Vanillinmandelsäure („VMS“) und die 5-Hydroxyindolessigsäure (,5-HIE“) wurden zahlreiche Methoden entwickelt, die sich verschiedener chromatographischer Methoden, der Hochspannungselektrophorese oder unterschiedlicher Elutionstechniken bedienen (1-22). Dagegen wurden Dihydroxyphenolsäuren bisher weniger untersucht $(23-29,19$, 30,31). Als chromatographische Methode mit dem geringsten Zeitaufwand hat sich die von STAHL (32) entwickelte Dünnschichtchromatographie (DC) erwiesen. Mit diesem Verfahren wurden Trennungen der Vanillinmandelsäure, der Vanillinsäurè (,VS“), der Homovanillinsäure und der 5-Hydroxyindolessigsäure mit quantitativer bzw. semiquantitativer kolorimetrischer

1) D 29.
Bestimmung angegeben (33-38). Andere bịsher dünnschichtchromatographisch getrennte aromatische Säuren $(39,40)$ sind von geringem klinischen Interesse.

Bei den oben erwähnten Phenol- und Indolsäuren handelt es sich um Abbauprodukte biogener Amine, welche im Organismus als neurohumorale Überträgersubstanzen wirken. Die Amine entstehen durch Decarboxylierung der entsprechenden Aminosäuren. Die freigesetzten Wirkstoffe werden durch oxydative Desaminierung bzw. O-Methylierung in 3-Stellung des Benzolringes rasch inaktiviert (1, 41-47). In der vorliegenden Arbeit sollten das chromatographische Verhalten und die Färbeeigenschaften weiterer Phenol- und Indolsäuren untersucht werden. Dabei interessierte besonders, ob diese Substanzen mit den bisher bestimmten Metaboliten interferieren. Die Möglichkeit einer vermehrten Ausschei- dung bei Sympathikustumoren war zu prüfen. Weiterhin sollte versucht werden, eine quantitative Bestimmungsmethode für die 5-HIE mit dünnschichtchromatographischer Trennung zu entwickeln, da bei der üblichen kolorimetrischen Technik nach UDENFRIEND (48) bzw. ihren Modifikationen (49) Störungen durch andere Metaboliten beschrieben wurden $(50-52)$. 


\section{Methodik}

Glasplatten $(20 \times 20 \mathrm{~cm})$ werden mit der Sorptionsmasse in einer Dicke von $0,25 \mathrm{~mm}$ mit Hilfe des „DESAGA"-Streichgerätes (Grundausrüstung Nr. 600) beschichtet.

Sorbentien: Kieselgel G Fa. „Merck“ bzw. Kieselgur G Fa. „Merck“ für Dünnschichtchromatographie nach SrakL.

\section{Vorbereitung der Platten zur Dünnschichtchromatograpbie:}

1. Jeweils 5 Platten mit einem Gemisch aus $30,0 \mathrm{~g}$ Kieselgel $\mathrm{G}$ mit $60 \mathrm{ml}$ aqua dest. (etwa $1 \mathrm{Min}$. gut durchschütteln) beschichten und etwa $30 \mathrm{Min}$. bei $100^{\circ}$ trocknen.

2. Jeweils 5 Platten mit Kieselgel $G$ und Kieselgur $G$ im Verhältnis 1:1 (je $25 \mathrm{~g}$ ) unter Zusatz von $2,5 \mathrm{~g}$ Leuchtpigment $\mathrm{ZS}$ super $\mathrm{Fa}$. „Riedel de $\mathrm{Haën}^{\text {“ }}$ und $90 \mathrm{ml}$ aqua dest. beschichten, 1 Std. bei etwa $100^{\circ}$ trocknen und 12 Stdn. im Exsikkator aufbewahren.

\section{Harnextraktion}

$10-20 \mathrm{~m} l$ Sammelurin mit konz. $\mathrm{HCl}$ auf $\mathrm{pH} 1$ ansäuern, mit $\mathrm{NaCl}$ sättigen und $3 \mathrm{mal} \mathrm{mit} \mathrm{je} 50 \mathrm{ml} /$ Diäthyläther p. a. bzw. $3 \mathrm{mal}$ mit je $40 \mathrm{ml}$ Essigsäureäthylester p. a. durch gründliches Ausschütteln extrahieren; Extrakte im Wasserbad eindampfen. Auflösen des Rückstandes in $1 \mathrm{~m} /$ A thanol bzw. Ester bei Kühlung auf $0^{\circ} \mathrm{im}$ Eiswasserbad. Auftragen der Harnextrakte mittels einer graduierten Spezialpipette, anschließend aufsteigende Chromatographie.

\section{Fließmittelsystemse}

Routinemäßig wurde das alkalische Steigmittelsystem Isopropanol/ Essigsäureäthylester/Ammoniak/Wasser im Verhältnis 45:30:17:8 (Fließmittelgemisch I, aufsteigende Chromatographie bei Normalsättigung) angewandt. Als Fließmittelsystem II wurde anschließend, nach Zwischentrocknen, Benzol/Eisessig im Verhältnis 90:10 (zweimalige aufsteigende Chromatographie bei Kammersättigung) verwendet. Weitere Steigmittelgemische sind bei den Ergebnissen aufgeführt.

\section{Lokalisation der Metaboliten}

Die einzelnen Substanzen wurden bei Verwendung von Fluoreszenz-Platten unter dem UV-Licht (Hanau-Lampe) bei $254 \mathrm{~m} \mu$ lokalisiert. Hierfür waren in der Regel (Hanau-Lampe) $5 \mu \mathrm{g}$ der Substanz erforderlich. Als Sprübreagenzien wurden meist verwendet: 2,6-Dichlorchinon-4-chlorimid (0,1\% in Methanol) bzw. diazotiertes p-Nitranilin ( $5 \mathrm{ml}$ der 0,1-proz. Lösg., $5 \mathrm{ml}$ 0,2-proz. Lösg. von Natriumnitrit in aqua dest., $1 \mathrm{ml} 1 \mathrm{~N} \mathrm{NaOH}$, nach dem Mischen sofort zu verwenden). Die Nachweisgrenze für Substanzen, die sich damit färbten, lag in der Regel bei 0,1 $\mu \mathrm{g}$. Weitere Sprühreagenzien sind in den Ergebnissen aufgeführt. Die Nachweisgrenze betrug hier meist $0, \dot{5}-2 \mu \mathrm{g}$.
Quantitative Bestinumung $(34,38)$

Bandförmiges Auftragen der eingeengten Extrakte, meist $150 \mu \mathrm{l}$, Entwicklung in den Fließmittelgemischen I und II, Lokalisation der Metaboliten anhand der Leitchromatogramme mit Reinsubstanzen, sowie Extrakt mit Zusatz von Reinsubstanzen unter dem UV-Licht, anschließend Färbung der Leitchromatogramme mit Dichlorchinonchlorimid, Abtragen der metabolithaltigen Zonen des nicht gefärbten Mittclfeldes in spitzkonige Zentrifugengläser, Elution mit $7 \mathrm{ml}$ Titrisolpuffer $\mathrm{pH} 1$, zentrifugieren, Einsatz von jeweils $5 \mathrm{ml}$ Uberstand zur Farbreaktion mit diazotiertem pNitranilin. Ablesen der Extinktion bei $510 \mathrm{~m} \mu$ im Zeiß „Elko II“ bei $2 \mathrm{~cm}$ Schichtdicke. Bei der Bestimmung der HVS wurde die Metabolit-haltige Zone in aqua dest. eluiert, die Farbreaktion mit Pauly's Reagenz vorgenommen. Das vorteilhaftere Rose'sche Reagenz $(38,42)$ wird bisher kommerziell nicht hergestellt und stand hier nicht zur Verfügung.

\section{Ergebnisse}

Steighöhen und Farbreaktionen der untersuchten Substanzen sind in Tabelle 1 aufgeführt. Phenylessigsäure sowie $\alpha$ - und $\beta$-Phenylmilchsäure waren mit Dichlorchinonchlorimid nicht färbbar und schieden somit als Störsubstanzen der interessierenden Metaboliten aus. Eine eindeutige Lokalisation auf Kieselgel-Platten nach aufsteigender Chromatographie im Fließmittelgemisch I, Besprühen der Platten mit Fluoreszein-Lösung und Betrachten unter der Wood'schen Lampe wurde nicht erreicht. Die chromatographische Entwicklung der Phenylmilchsäuren war möglich bei Verwendung von $100 \mathrm{ml}$ einer 8-proz. NaCl-Lösung mit Zusatz von $1 \mathrm{ml}$ Eisessig, einem Fließmittelgemisch, das bisher zur papierchromatographischen Trennung anderer Phenol- und Indolsäuren benutzt worden war (53). Die $R_{\mathrm{F}}$-Werte betrugen etwa 0,7 bzw. 0,8. In wassergesättigtem Butanol/ Eisessig (98:2) wurden $R_{\mathrm{F}}$-Werte von etwa 0,4 gefunden. Nach Besprühen mit Phenylhydrazinhydrochlorid in 45-proz. $\mathrm{H}_{2} \mathrm{SO}_{4}$ mit anschließender halbstündiger Erhitzung der Platte bei $100^{\circ}$ gab $\beta$-Phenylmilchsäure eine rote, $\alpha$-Phenylmilchsäure eine blauviolette Fluoreszenz unter der Hanau-Lampe.

Phenylbrenztraubensäure konnte mit Fließmittelgemisch I entwickelt werden (Tab. 1). Der sonst übliche

Tab. 1. Steighöhen (in $\mathrm{cm}$ ) und Farbreaktionen von Phenol- und Indolsäuren mit Dichlorchinonchlorimid

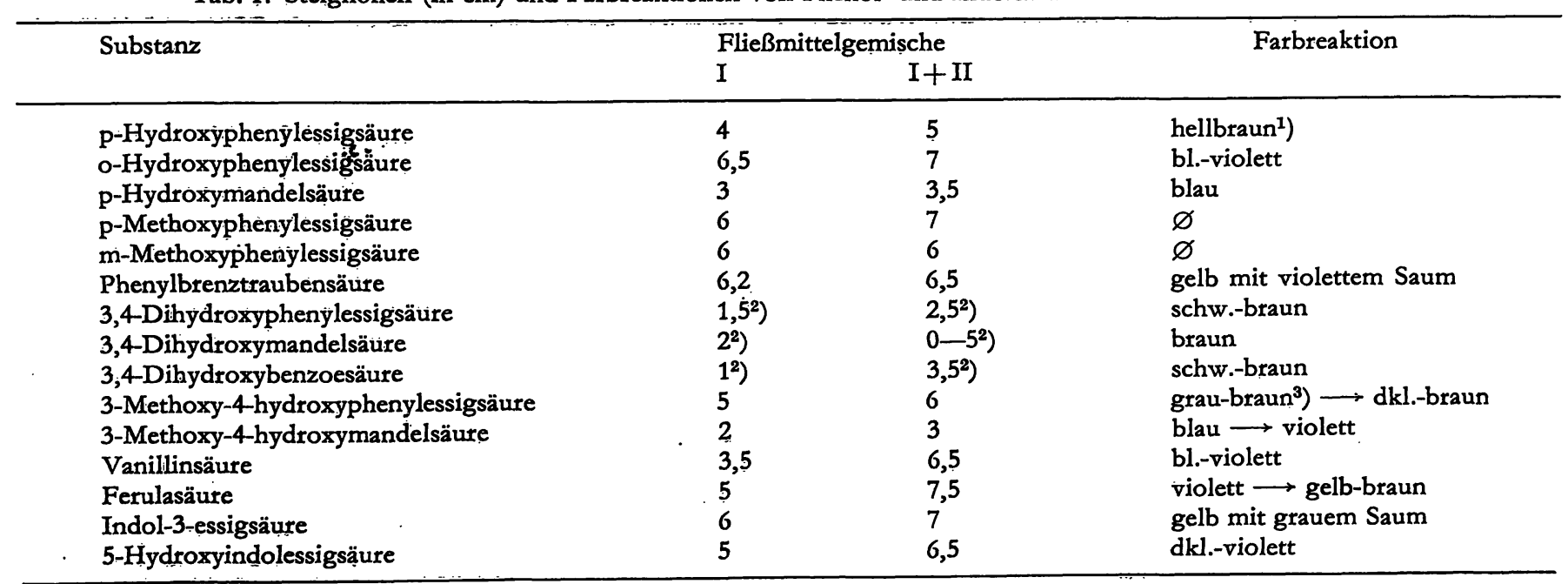

1) Bei Besprühen schwach alkalischer Kieselgel-Platten.

2) Schweifbildung; spontane Braunfärbung bei Entwicklung mit alkalischen Fließmitteln.

3) Auftreten der Farbreaktion $1 / 2-1$ Std. nach Besprüuhhen. 
Nachweis mit Ferrichlorid und anschließendem Alkalisieren mit $\mathrm{NaOH}$ ergab auf Kieselgel-Platten eine schlecht verwertbare Farbreaktion. Eine Blaufärbung wurde durch Besprühen mit Phosphorsäure + Phosphormolybdänsäure und anschließendem kurzen Erhitzen erzielt. Bei Besprühen mit Phenylhydrazinhydrochlorid und halbstündigem Erhitzen bei $100^{\circ}$ trat eine Blaufluoreszenz bei Betrachtung unter der Hanau-Lampe auf.

3-Methoxy- bzw. 4-Methoxy-phenylessigsäure ergaben mit Dichlorchinonchlorimid keine Farbreaktion und waren nur anhand der Fluoreszenzlöschung zu lokalisieren. p-Hydroxyphenylessigsäure färbte sich mit Dichlorchinonchlorimid blau, wenn die DC-Platten sofort nach der Entnahme aus dem alkalischen Fließmittelgemisch besprüht wurden, während o-Hydroxyphenylessigsäure auch nach Chromatographie im sauren Fließmittelgemisch bei Besprühen mit Dichlorchinonchlorimid und Alkalisieren eine Sofortreaktion ergab. Eine weitere empfindliche Nachweismöglichkeit bestand darin, daß die mit dem alkalischen Fließmittelsystem entwickelte DC-Platte 15 Minuten einer starken UVStrahlung (Hanau-Höhensonne) ausgesetzt wurde (Abb. 1). Bereits 0,1-0,5 $\mu \mathrm{g}$ p-Hydroxyphenylessigsäure verfärbten sich graubraun, während andere MonoOxyphenolsäuren und die 5-HIE bei dieser photochemischen Reaktion erst in Konzentrationen von $1-5 \mu \mathrm{g}$ sichtbar wurden. Nach UV-Bestrahlung war die p-Hydroxyphenylessigsäure bei Betrachtung unter der Hanau-Lampe neben anderen Substanzen als fluoreszierender Fleck abzugrenzen. Fluoreszenz und Braunfärbung traten bei Konzentration über $1 \mu \mathrm{g}$ p-Hydroxyphenylessigsäure auch bei Trennung mit den Fließmittelsystemen I und II auf. Gegenüber der Farbreaktion mit

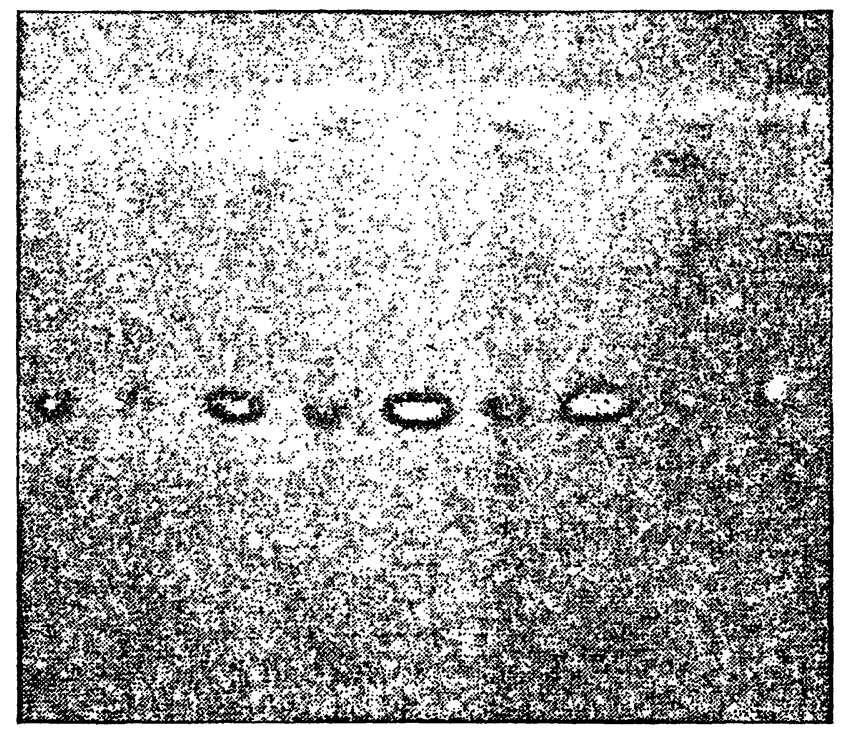

Abb. 1

Dünnschichtchromatogramm

Entwicklung mit Fließmittclgemisch I

Aufgetragen sind 2,5-, 5-, 7,5 und $10 \mu \mathrm{g}$ p-Hydroxyphenylessigsäure, sowie 2,5- 5, 7,5 und $10 \mu l$ Harnextrakt (Sammelharn nach oraler Tyraminbelastung) und $5 \mu l$ 'Extrakt mit Zusatz von $2 \mu \mathrm{g}$ pHydroxyphenylessigsäure (letzte Kolonne); 15 Min. Bestrahlung mit UV-Licht ohne anschließende Färbung der DC-Platte
Dichlorchinonchlorimid bzw. diazotiertem p-Nitranilin bestand der Vorteil, daß die p-Hydroxyphenylessigsäure nach Einwirkung von UV-Licht von anderen Metaboliten gut abgegrenzt werden konnte, welche in verschiedenen Fließmittelgemischen ähnliche Steighöhen aufwiesen. Die Methode wurde orientierend bei Versuchen mit Tyrosin- und Tyraminbelastung beim Menschen angewandt.

Dihydroxyphenolsäuren wurden in den Fließmittelgemischen I bzw. I + II wegen ihrer hohen Polarität kaum entwickelt (Tab. 1). Außerdem bewirkte Alkali eine Zersetzung, so daß sich braunschwarze Flecken mit Schweif in der Nähe des Startpunktes bildeten. Eine Entwicklung der Dihydroxyphenolsäuren gelang mit dem Fließmittelgemisch Butanol (wassergesättigt)/Eisessig (98:2); die $R_{\mathrm{F}}$-Werte lagen für Dihydroxyphenylessigsäure bei $0,65-0,75$, für Dihydroxymandelsäure bei 0,25-0,35. Eine Entwicklung der Flecken mit Sprühreagenzien war nicht erforderlich; Einwirkung von Ammoniak bewirkte eine selektive Braunfärbung. Bei Entwicklung der 3-Methoxy-4-Hydroxyphenolsäuren im Fließmittelgemisch I war die Trennung von anderen Metaboliten nach $10 \mathrm{~cm}$ Laufstrecke der Fließmittelfront ungenügend. Wurde die Laufstrecke auf $15-17 \mathrm{~cm}$ erhöht, so verbesserte sich die Trennung. Die VS saß jedoch der VMS dicht auf. Die HVS, mit dem höchsten $R_{\mathrm{F}}$-Wert im alkalischen Fließmittelgemisch, war, vor allem bei höheren Konzentrationen, an ihrem färberischen Verhalten $z u$ erkennen. Eine gute Trennung dieser Substanzen voneinander und von anderen Metaboliten in Harnextrakten wurde erreicht, wenn sie auf Kieselgel-Kieselgur-Platten nacheinander mit den Fließmittelgemischen I und II entwickelt wurden (Tab. 1).

Bei umgekehrter Reihenfolge, d.h. bei Anwendung des sauren Gemisches als erstem und des alkalischen als zweitem Steigmittel, verschmierten die metabolithaltigen Zonen. Wurde die zweimalige aufsteigende Chromatographie mit dem Fließmittelgemisch Benzol/ Eisessig allein vorgenommen, so blieb die VMS in der Nähe des Startpunktes sitzen. Erhöhte man die Polarität durch Zusatz von Methanol (Fließmittelgemisch Benzol/Methanol/Eisessig = 90:15: $10)$, so war die Trennung für semiquantitative Bestimmungen ausreichend.

Ferulasäure war hier im Gegensatz zur kombinierten Chromatographie mit Fließmittel I und II von der VS schlecht abzugrenzen, unterschied sich jedoch im färberischen Verhalten: Beim Besprühen trat Violettfärbung auf, die rasch zu einer gelbbraunen Färbung mit violettem Ring abblaßte. Die Erhöhung des Methanolanteils im sauren Fließmittelgemisch bewirkte ein Schmieren der VMS-Flecke und eine schlechte Trennung von Substanzen, die im unteren Drittel der DC-Platten lokalisiert waren. Die Verwendung von Isopropanol anstelle von Methanol führte zu gleichen Resultaten. Wurde Benzol als nichtpolare Komponente durch Chloroform ersetzt, so schmierten ebenfalls zahlreiche untersuchte Substanzen.

5-HIE ließ sich auf Grund der Steighöhe in Fließmittelgemisch I allein nicht sicher von anderen Harnmetaboliten abgrenzen. Kein Harnmetabolit, der aus angesäuertem Harn mit Äther bzw. Essigsäureäthylester extrahiert wurde und eine vergleichbare Steighöhe hatte, wies jedoch die tiefdunkle Violettfärbung mit Dichlorchinonchlorimid und eine Rotbraunfärbung bei $\mathrm{Be}$ sprühen der DC-Platten mit diazotiertem p-Nitranilin 
auf. Bei kombinierter Chromatographie im Gemisch I und II war die 5-HIE unterhalb einer breiten UV-absorbierenden Zone eines unbekannten Harnmetaboliten zu lokalisieren, der keine Farbreaktion mit Dichlorchinonchlorimid und diazotiertem p-Nitranilin ergab, sowie dicht oberhalb eines anderen färbbaren Metaboliten.

\section{Klinische Anwendung}

Die semiquantitative Bestimmung der p-Hydroxyphenylessigsäure in fraktioniert gesammelten Harnproben wurde bei 3 Probanden nach oraler Zufuhr von $100-500 \mathrm{mg}$ l-Tyrosin und bei 3 Versuchspersonen nach Gabe von $40-60 \mathrm{mg}$ Tyramin angewandt. Nach Zufuhr von Tyrosin kam es nur zu einer geringfügigen Zunahme der Phenolsäure im Harn, selbst bei Gabe von $500 \mathrm{mg}$ Tyrosin. Nach Ingestion von Tyramin trat eine rasch einsetzende Mehrausscheidung von $\mathrm{p}$-Hydroxyphenylessigsäure aut, die in der 2. oder 3. 4-Stundensammelperiode das Maximum erreichte. Die Rückgewinnung von Tyramin in Form der „freien“ Phenolsäure lag zwischen 7 und $20 \%$.

Bei der angewandten Extraktion und dünnschichtchromatographischen Trennung konnten wir bei Gesunden keine nennenswerten Mengen von Dihydroxyphenolsäuren, im Harn nachweisen (Abb. 2). - Erhöhte Mengen waren in 5 von 12 Fällen mit Phaeochromozytom, sowie bei 2 Erwachsenen mit malignen Sympathikustumoren wahrscheinlich. Zusätżliche Trennungen zur Differenzierung in Dihydroxymandelsäure und Dihydroxyphenylessigsäure wurden nicht vorgenommen. Bei 3 von 4 Kindern mit Neuroblastom waren hohe Konzentrationen nachzuweisen (Abb. 3).

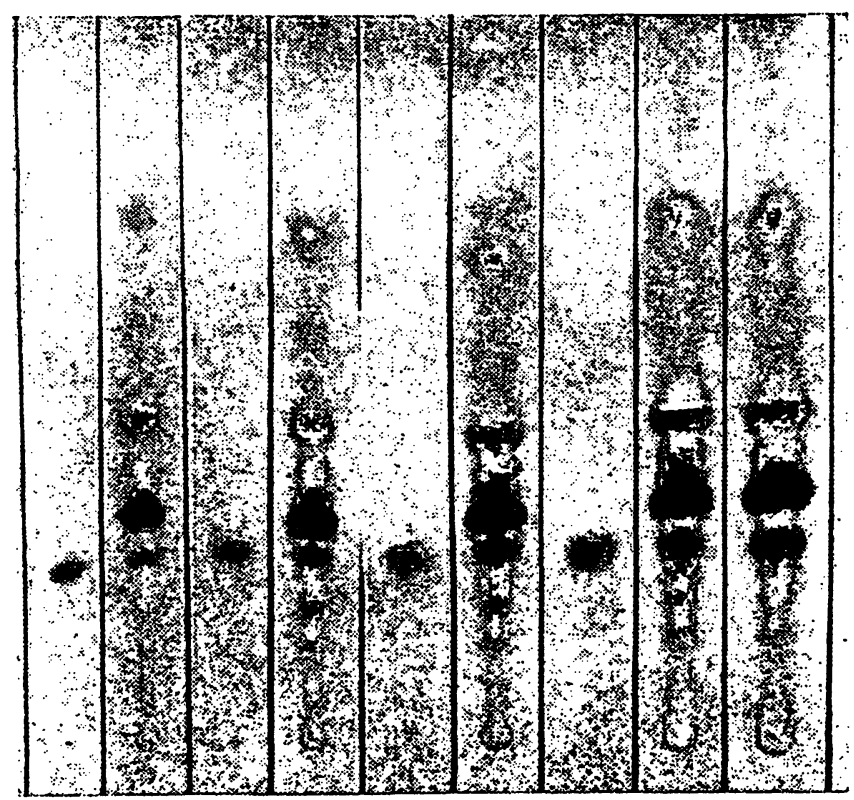

Abb. 2

Dünnschichtchromatogramm eines Harnextraktes (Phaeochromozytomverdacht)

Aufgetragen sind 0,25-1 $\mu \mathrm{g}$ VMS, 2,5-10 $\mu l$ des $20: 1$ konzentrierten Harnextraktes, sowie $10 \mu /$ Extrakt mit Zusatz von $1 \mu \mathrm{g}$ VMS (letztes Feld). VMS nicht vermehrt, Dihydroxysäuren in geringer Konzentration am Startpunkt
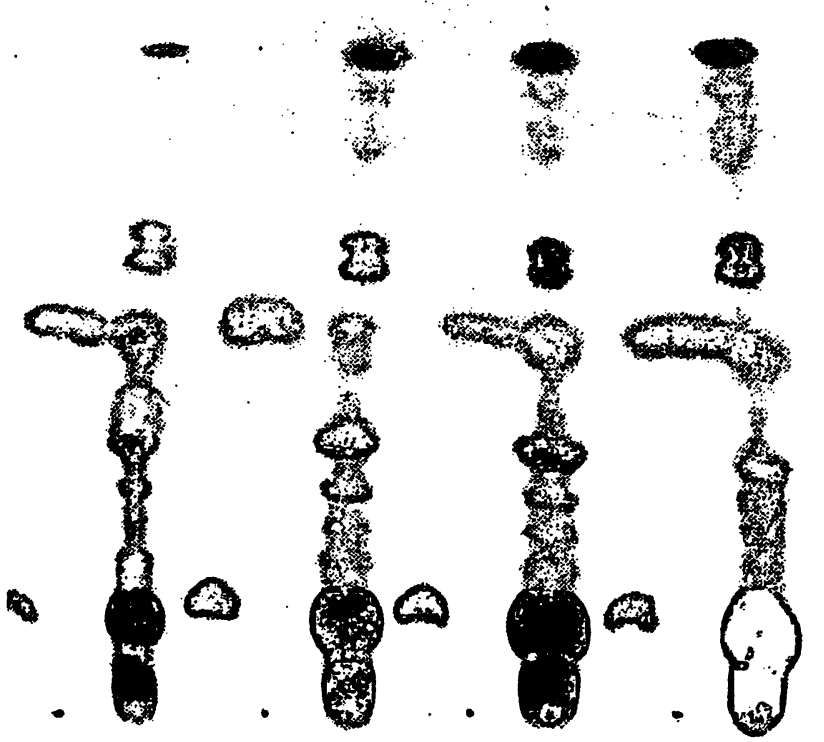

Abb. 3

Dünnschichtchromatogramm, Harn eines Kindes mit Neuroblastom

Entwicklung: Fließmittelgemisch I +2 mal Fließmittelgemisch II, Farbreaktion mit Dichlorchinonchlorimid. Aufgetragene Standards: $2,5-10 \mu \mathrm{g}$ VMS, 5-40 $\mu \mathrm{g}$ HVS, 5-40 $\mu \mathrm{g}$ VS. Vom Harnextrakt (20:1 konzentriert) sind 2,5-10 $\mu$ l aufgetragen. Man erkennt die erhöhten Konzentrationen an Katecholaminmetaboliten. Die den Startpunkten des Extraktes aufsitzenden Flecken wurden im System Butanol (wassergesättigt) / Eisessig als Dihydroxyphenylessigsäure identifiziert. Bei mehreren Flecken handelt es sich wahrscheinlich um Monohydroxyphenolsäuren, die ebenfalls in erhöhten Konzentrationen vorhanden sind

Nach Trennung im Fließmittelsystem Butanol/Eisessig entwickelten sich bei Bedampfen der DC-Platten mit Ammoniak dunkelbraune Flecken, deren Lokalisation der Dihydroxyphenylessigsäure entsprach. Mengenberechnungen erfolgten nicht, da die untersuchten Dihydroxysäuren wenig stabil sind und aus dem Harn in die Esterphase nur unvollständig extrahiert werden.

Die HVS war $6 \mathrm{mal}$ bei malignen Sympathikusgeschwülsten auf mehr als $5 \mathrm{mg} / 24 \mathrm{Stdn}$. erhöht, jedoch bei keinem der 12 Phaeochromozytomfälle. Die Tagesmengen der VMS lagen bei den 16 Patienten mit Sympathikustumoren zwischen 8 und $142 \mathrm{mg} / 24 \mathrm{Stdn}$. Werte der „endogenen" VS von mehr als $5 \mathrm{mg} / 24 \mathrm{Stdn}$. fanden wir $3 \mathrm{mal}$ beim Neuroblastom (Abb. 3), einmal bei einem malignen Glomustumor. - Im Gegensatz zu den Sympathikustumoren lagen die Werte der sauren Katecholaminmetaboliten, insbesondere die der VMS, bei 10 Fällen mit Carcinoidsyndrom stets im Normbereich und betrugen $2,5-5 \mathrm{mg} / 24 \mathrm{Stdn}$., während die Werte der 5-HIE zwischen 30 und $200 \mathrm{mg} / 24 \mathrm{Stdn}$. lagen (Abb. 4).

Die Möglichkeit der gleichzeitigen quantitativen Bestimmung von VMS, HVS, VS und 5-HIE wurde am Beispiel der pharmakologischen Beeinflussung des Katecholamin- und Serotoninstoffwechsels durch Prenylamin (,Segontin“) geprüft. Die Wiederfindung der zum Harn zugesetzten Metaboliten bzw. die Rückgewinnung 


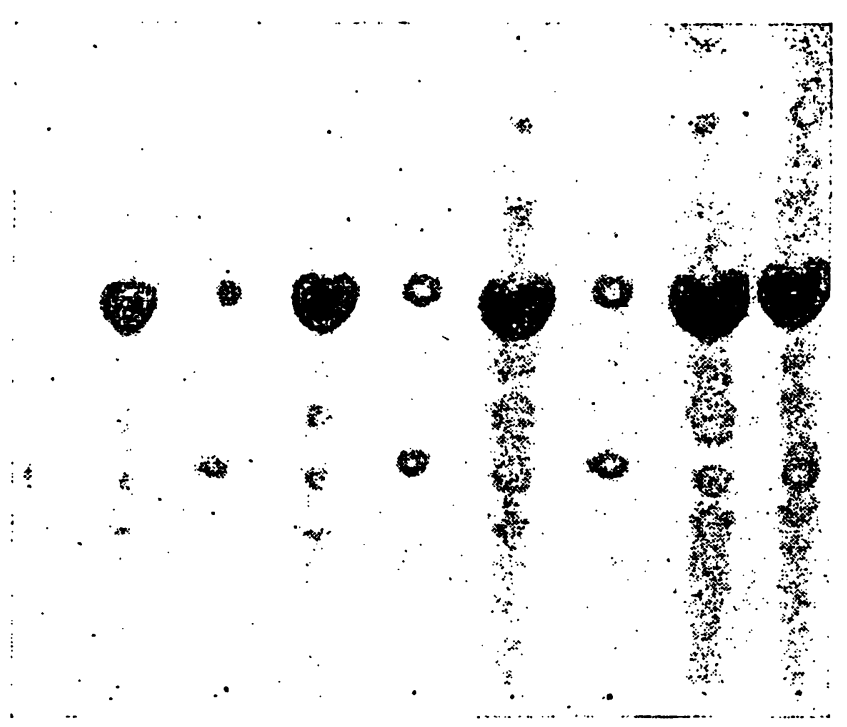

Abb. 4

Dünnschichtchromatogramm: Harnextrakt eines Patienten mit Carcinoidsyndrom

Entwicklung im Fließmittelgemisch I, Färbung mit diazotiertem p-Nitranilin. Als Standard sind aufgetragen 0,25-1 $\mu \mathrm{g}$ VMS bzw. 5-HIE, sowie 2,5-10 $\mu$ l Extrakt. Die Konzentration der VMS im Harnextrakt ist normal, die der 5-HIE stark erhöht

aus Chromatogrammen mit der Reinsubstanz war für HVS und VS annähernd quantitativ; für die VMS betrug der Vetlust im Mittel 25\%, für die 5-HIE etwa 20\%. 5 gesunde Probanden nahmen im subakuten Versuch $3 \mathrm{mal}$ täglich $60 \mathrm{mg}$ Prenylamin per os; 3 Klinikpatienten erhielten bei einmaliger Belastung $180 \mathrm{mg}$ als Einzeldosis. Die VMS stieg bei 4 der 5 gesunden Probanden am ersten Tag der Segontinzufuhr über die Ausgangswerte an; im 5. Fall kam es am 3. und 4. Tag der Medikamenteneinnahme zu einer Mehrausscheidung des Katecholaminmetaboliten. Ein Abfall der in Tagund Nachtharn getrennt bestimmten VMS-Mengen bei weiterer Zufuhr von Prenylamin über 5 bis 7 Tage war nicht zu sichern; in einem Fall wurde sogar bei Versuchsende nochmals ein hoher VMS-Wert gefunden. Bei 3 Klinikpatienten führte Segontin zu einer erheblichen Mehrausscheidung der VMS im Harn (Abb. 5). Die Ausgangswerte lagen hier im allgemeinen niedriger als bei gesunden berufstätigen Probanden.

Der Dopamin-Metabolit HVS wurde bei 2 Gesunden über eine Dauer von 13 Tagen untersucht. Eine sichere, auf das Medikament zu beziehende Vermehrung konnten wir nicht beobachten. Die Beurteilung der VSAusscheidung wurde dadurch erschwert, daß Nichtbeachten der Diätvorschriften zu einer erheblichen Mehrausscheidung von „exogener" VS führen kann; Werte von $150-180 \mu \mathrm{g} / \mathrm{Std}$. in Einzelportionen traten bei Gesunden vor allem nach Genuß vanillehaltiger Süßspeisen oder Kuchen auf. Bei 3 von 5 Gesunden blieb die Ausscheidung von VS unter Zufuhr von Prenylamin etwa konstant; in 2 Fällen kam es zu einem anhaltenden Anstieg trotz Beachtung der Diätvorschriften. Der bei einem der 3 Klinikpatienten beobachtete Anstieg der VS (Abb.5) in einer Harnportion ist wahrscheinlich

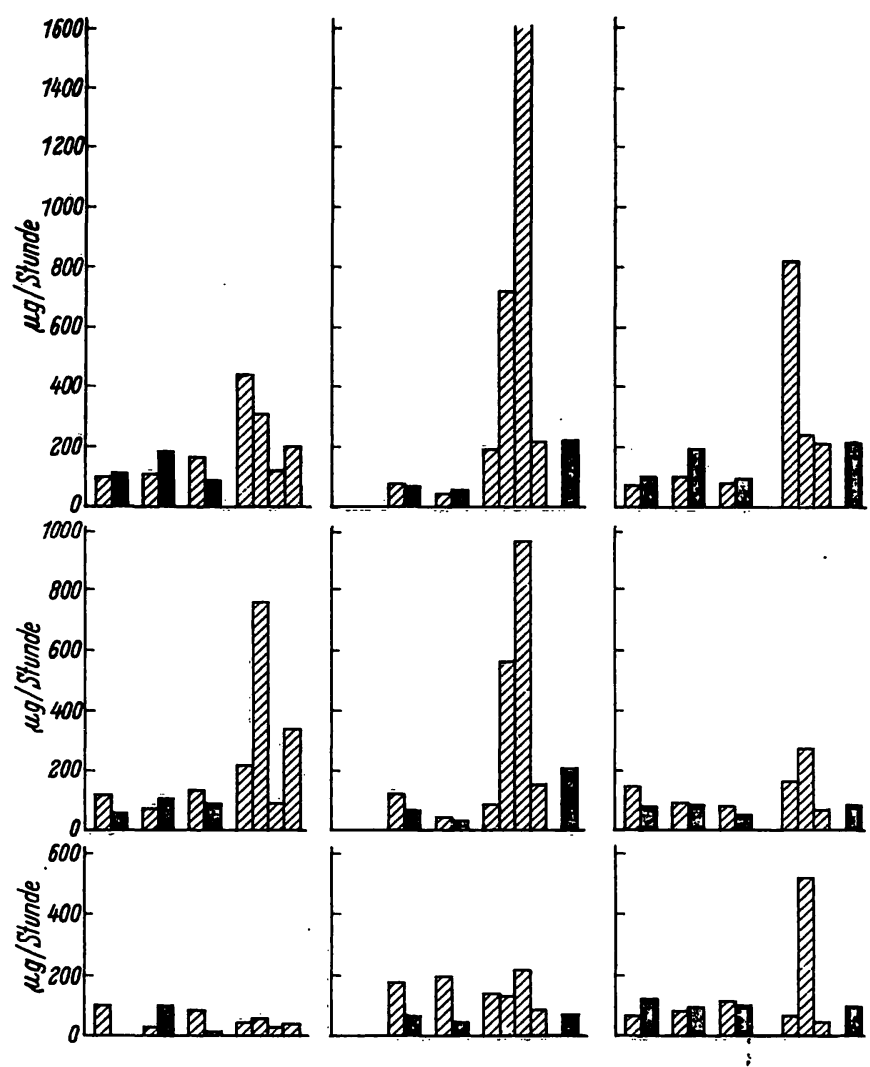

Abb. 5

Ausscheidung von 5-HIE (oben), VM̈S (Mitte) und VS (unten) bei 3 Klinikpatienten vor und nach Belastung mit $180 \mathrm{mg}$ Segontin per os

Schraffierte Säulen $=$ Tagesharn; schwarze Säulen $=$ Nachtharn

ebenfalls durch exogene, zur VS metabolisierte Substanzen bedingt.

Die mit dünnschichtchromatographischer Trennung und Koppelung an diazotiertes $\mathrm{p}$-Nitranilin bestimmten Werte der 5-HIE lagen bei 4 Gesunden und 3 Klinikpatienten im Normbereich zwischen 2 und $10 \mathrm{mg} /$ $24 \mathrm{Stdn}$. (49). In einem Fall war die 5-HIE-haltige Zone wegen der Úberlagerung durch andere Metaboliten chromatographisch nicht abzugrenzen. Nach $180 \mathrm{mg}$ Prenylamin stieg in allen Fällen am ersten Belastungstag die Menge des Serotonin-Metaboliten an, ohne jedoch den Normbereich zu überschreiten (Abb. 5). Dieser Segontin-Effekt ist mit der Wirkung von Reserpin vergleichbar, für das eine Liberierung des Amins und eine exhöhte 5-HIE-Ausscheidung seit langem bekannt sind. Setzte man die Zufuhr von Prenylamin über 5-9 Tage fort, so sank die 5-HIE-Eliminierung auf das Ausgangsniveau oder etwas darüber liegende Werte ab.

\section{Diskussion}

Verschiedene Fließmittelsysteme, die Verwendung mehrerer Sorptionsmaterialien und die Einbeziehung der Fluoreszenzlöschung, sowie der photochemischen Umsetzung im UV-Licht neben Standard-Farbreaktionen gestatten es, zahlreiche Phenol- und Indolsäuren dünnschichtchromatographisch zu trennen und zu identifizieren. Mit den verwendeten Testsubstanzen wird das 
„Phenolsäuremuster" des menschlichen Harns $(1,54,55)$ zwar nicht vollständig entschlüsselt, für diagnostische Zwecke kann man sich jedoch auf wenige Metaboliten beschränken, da die Mehrzahl der Phenolkörper aus der Nahrung oder der intestinalen Darmflora stammt. Diese „exogenen" Phenolsäuren sind daran zu erkennen, daß ihre Ausscheidung im Harn stark schwankt und bei Nahrungskarenz bzw. mehrtägiger Ernährung mit Kohlenhydraten rasch abfällt $(56,57)$.

Als bisher nicht näher untersuchten Metaboliten einer aromatischen Aminosäure prüften wir in orientierenden Versuchen das Verhalten der p-Hydroxyphenylessigsäure. Mit der hier erstmals beschriebenen photochemischen Umwandlung dieses Tyramin-Metaboliten wurde ein neues Nachweisverfahren entwickelt, das eine Identifizierung gegenüber anderen Substanzen mit ähnlichem chromatographischen Verhalten gestattet. In den untersuchten Harnextrakten war die p-Hydroxyphenylessigsäure die einzige Substanz, die sich bei entsprechender Steighöhe unter UV-Licht braun färbte. Überraschenderweise wurde 1-Tyrosin nach oraler Zufuhr nur mit einem Anteil von weniger als $10 \%$ zur freien Phenolsäure umgesetzt. Dieser Wert liegt erheblich niedriger als die Auffindung von 5-HIE bei Belastungsversuchen mit der heterocyclischen Aminosäure 5Hydroxytryptophan (58). Tyramin wurde rasch desaminiert; das Ausscheidungsmaximum der p-Hydroxyphenylessigsäure im Harn trat. nach oraler Tyraminzufuhr innerhalb von 2-4 Stunden auf. Auch hier war die Wiederfindung des umgesetzten Amins in Form der nicht konjugierten Phenolsäure, verglichen mit der Elimination der 5-HIE bei Serotoninbelastungsversuchen, gering (58). Dieser Befund überrascht, da Tyramin als bevorzugtes Substrat der Monooxydase gilt und auch bei parentaler Zufuhr - nach der kurzen Blutdruckwirkung zu urteilen - rasch abgebaut wird. Nach Säurehydrolyse der Harnproben fanden wir im allgemeinen keine höheren Konzentrationen, so daß eine Koppelung der p-Hydroxyphenylessigsäure wahrscheinlich von untergeordneter Bedeutung ist. Dagegen muß offen bleiben, ob ein bakterieller Abbau des Tyramin im Darmtrakt zu anderen Metaboliten mitverantwortlich für die geringe Wiederfindung ist.

Für die Analyse hormonal akțiver Tumoren des sympathiko-adrenalen Systems wurde das Spektrum untersuchter saurer Aminmetaboliten von der Vanillinmandelsäure auf Homovanillinsäure, Dihydroxyphenylessigsäure, Dihydroxymandelsäure und Vanillinsäure erweitert. Zur Diagnose des histologisch reifen, nicht metastasierenden Phaeochromocytoms teicht die semiquantitative Bestimmung der VMS auf Grund unserer an 12 Fällen gewonnenen Erfahrung aus. Beim Neuroblastom, einer im Kindesalter auftretenden malignen Sympathikusgeschwulst, und bei malignen Sympathikustumoren des Erwachsenen ist die Steigerung des Umsatzes biogener Amine nicht auf Noradrenalin und Adrenalin beschränkt. Vorstufen der Hormone, z. B. Dopamin und seine Abbauprodukte, vor allem die HVS, werden vermehrt im Harn ausgeschieden.
Unsere Befunde bei einem Erwachsenen mit malignem Glomustumor und bei vier Kindern mit Neuroblastom bestätigen die Ergebnisse früherer Untersucher $(9,13$, 14, 59-62). Die Vermehrung der VS in vier Fällen mit malignen Sympathikustumoren entspricht Befunden von Sturm (63) bzw. Robinson $(8,64)$ beim Phaeochromoblastom. Nach Dirscherl und Mitarbeitern (42) sowie GoODALL und ROSEN (46) stammen kleine VS-Mengen aus dem Katecholaminstoffwechsel, wahrscheinlich als Abbauprodukt der VMS. Von Interesse sind Einzelbeobachtungen einer erhöhten Ausscheidung der Vanillinmilchsäure (59) sowie von Protokatechualdehyd (65) bei malignen Sympathikustumoren. Theoretisch könnten beide Substanzen zur VS umgesetzt werden. Es stellt sich daher die Frage, ob die „endogene" VS unter physiologischen oder pathologischen Bedingungen nicht ausschließlich aus VMS, sondern auch aus anderen Vorstufen entsteht.

9 von 10 Fällen mit metastasierendem Argentaffinom wiesen das Vollbild des Carcinoidsyndroms und eine erhebliche Steigerung des Serotoninstoffwechsels, kenntlich an einer vermehrten Oxyindolessigsäure-Ausscheidung im Harn, auf. Im Gegensatz zu v. STudNITz (66) war der Katecholaminstoffwechsel bei unseren Fällen normal; nur bei einer Patientin mit klinisch und biochemisch „stummen" malignem Carcinoid lag die VMS geringfügig über der oberen Normgrenze. Wir glauben daher nicht, daß eine erhöhte Freisetzung und Umsetzung von Katecholaminen für die Pathophysiologie des Carcinoidsyndroms bedeutsam ist.

Unsere Untersuchungen über den Einfluß des Coronardilatators Segontin auf den Aminstoffwechsel lassen schließlich erkennen, $d a ß$ die angewandten dünnschichtchromatographischen Trennverfahren sich nicht nur für diagnostische Fragen eignen, sondern auch zur Wirkungsanalyse von Arzneimitteln am Menschen beitragen.

Vom Tierexperiment sind mehrere pharmakologische Wirkungsqualitäten bekannt $(67,68,69)$ : Biogene Amine, insbesondere Noradrenalin, Adrenalin und Serotonin werden aus der Speicherform freigesetzt; der Gewebsspiegel der Amine sinkt ab. Gleichzeitig wird die erregende Wirkung der endogen freigesetzten Uberträgerstoffe am Erfolgsorgan blockiert. Beim Menschen war bisher nicht geklärt, ob einer Phase vermehrter Ausscheidung biogener Amine und ihrer Metaboliten im Harn $(70,71)$ bei längerer Anwendung des Präparates eine Verminderung von Metaboliten des Katecholamin- und Serotoninstoffwechsels folgt (72).

Unsere Befunde bestätigen die Ergebnisse von KusCHKE (71), wonach die Ausscheidung der 3-Methoxy-4-Hydroxymandelsäure zu Beginn der Prenylaminbehandlung zunimmt. Dies entspricht einem vermehrten Abbau von Noradrenalin und Adrenalin im Organismus. Dopamin scheint bei der Aminliberierung weniger beteiligt zu sein, da die HVS kaum ansteigt. Die erhöhte Ausscheidung von 5-HIE ist auf eine Freisetzung von Serotonin zürückzuführen (70). Gesicherte Befunde, die für eine sekundäre Hemmung des Katecholamin- und SerotoninUmsatzes sprächen, ergaben sich aus unseren Versuchen nicht; bei Zufuhr des Pharmakons bis zu einer Woche sanken die VMS, HVS und 5-HIE nicht unter das Aus- 
gangsniveau ab. Ob der im Einzelfall beobachtete Spätanstieg der VMS und erhöht bleibende 5-HIE-Werte dem Pharmakon zuzuschreiben ist oder durch die spontane Periodik der Ausscheidung von Aminmetaboliten erklärt werden kann (38), müßte an größeren Reihen untersucht werden.

Schwierig zu deuten ist die in zwei Fällen bei längerer Einwirkung von Prenylamin beobachtete Zunahme der VS, offenbar unabhängig von den Diätgewohnheiten.
Für das $\alpha$-Methyldopa, ein blutdrucksenkendes Medikament mit zentraldämpfender Wirkung, wurde ein ähnliches, von der Ernährung unabhängiges Verhalten der VS beschrieben (73). Daher muß offen bleiben, ob Prenylamin andere, bisher unbekannte Angriffspunkte im Katecholaminstoffwechsel besitzt.

Die Untersuchungen wurden von der Deutschen Forschungsgemeinschaft gefördert. Frl. B. TeựFeL danken wir für fleißige und gewissenhafte Mitarbeit.

\section{Literatur}

1. Armstrong, M. D., A. McMillan und K. N. F. Shaw, Biochim. biophysica Acta (Amsterdam) 25, 422 (1955). - 2. BAjor, G. F. und N. G. Clark, J. Chromatograph. 14, 477 (1964). - 3. CowARD, R. F., P. Smith und O. S. Wirson, Clin. chim. Acta (Amsterdam) 9, 381 (1964). - 4. Flecher, R., J. D. Chandiey und S. Rosenblatt, Analyt. Biochem. (New York) 9, 54 (1964). - 5. GöDICKE, W. und K.H. Brosowskr, J. Chromatogr. 15, 88 (1964). 6. Jepson, J. B., Chromatographic techniques. Academic Press (London), (1958). - 7. Pisano, J. J., J. R. Crout und D. AbraHAM, Clin. chim. Acta (Amsterdam) 7, 285 (1962). - 8. Robinson, R., J. Ratcliffe und P. Sarth, J. clin. Path., London 12, 541 (1959). - 9. Ruthven, C. R. J. und M. SANDLER, Biochem. J. 83, 30 (1962); Analyt. Biochem. (New York) 8, 282 (1964). - 10. SAto, T., Y. WADA und M. MAEBASHI, Tohoku J. exp. Med. 80, 1 (1963). - 11. SEN, N. P. und P. L. MCGEeR, Biochem. biophysic. Res. Commun. 13, 5 (1963). - 12. Sharman, D. F., Brit. J. Pharmacol. Chemotherapy 20, 204 (1963). - 13. v. Srudnirz, W., Scand. J. Clin. Laborat. Invest. Suppl. 48 zu 12, 1 (1960). - 14. v. Studnitz, W., Klin. Wschr. 40, 163 (1962). - 15. v. Studnitz, W. und A. Hanson, Scand. J. Clin. Laborat. Invest. 11, 101 (1959). - 16. Sturm, jr., A., Clin. chim. Acta (Amsterdam) 7, 714 (1962). - 17. Sturm, jr., A. und H. W. SchejA, J. Chromatogr. 16, 194 (1964). - 18. SundermanN, W., P. D. Cleveland, N. C. Law und F. W. Sundermann, Amer. J. Clin. Path. 34, 293 (1960). - 19. WADA, Y., Tohoku J. exp. Med. 79, 389 (1963). - 20. WeILMalherbe, H., Analyt. Biochem. (New York) 4, 485 (1964); diese Z. 2, 161 (1964). - 21. WeISE, V. K., R. K. MCDonald und E. H. LA Brosse, Clin. chim. Acta (Amsterdam) 6, 79 (1961). - 22. Williams, C. M., A nalyt. Biochem. (New York) 4, 423 (1962). 23. Euler, Chr. v., U. S. v. Euler and I. Floding, Acta physiol. scand. Suppl. 118, 32 (1955). - 24. KLeIN, D. und J. M. CHERNaIK, Clin. Chem. (New York) 7, 257 (1961). - 25. LeNZ, H., Psychopharmacologia 3, 146 (1962). - 26. McMillaN, M., Lancet I, 715 (1957). - 27. MrYake, H., H. Yoshida und R. Imaizumi, Jap. J. Pharmacol. 12, 79 (1962). - 28. Rosengren, E., Acta physiol. scand. 49, 370 (1960). - 29. SOURkEs, T. L., R. L. DENton, G. F. Murphy, B. Chavez und Saint Cyr, Pediatr. 31, 660 (1963). - 30. Wada, Y. und N. Watanabe, Tohoku J. exp. Med. 84, 161 (1964). - 31. Williams, C. M. und R. H. Leonhard, Analyt. Biochem. (New York) 5, 362 (1963). - 32. Stakt, E., Dünnschichtchromatographie, ein Laboratoriumshandbuch. Springer-Verlag, Berlin-Göttingen-Heidelberg (1962). - 33. SANKOFF, I. und T. L. Sourkes, Canad. J. Biochem. Physiol. 41, 1381 (1963). -34. Schmid, E. und N. Henning, Klin. Wschr. 41, 566 (1963). 35. Schmid, E., L. Zicha, J. Krautheim und J. Blumberg, Med. exp.(Basel) 7,8 (1962). - 36. Segura-Cardona, R.und K. Soemring, Med. exp. (Basel) 10, 251 (1964). - 37. StaHL, E. und H. KALDEwaY, Hoppe-Seylex's Z. physiol. Chem. 323, 182 (1961). - 38.
Tautz, N. A., G. Voltmer und E. Schmid, Klin. Wschr. 43, 233 (1965). - 39. Pastuska, G., Z. analyt. Chem. 179, 355 (1961). 40. Pastuska, G. und H. J. Petrowirtz, Chemiker-Ztg. 86, 311 (1962). - 41. AxELrod, J., Science (Washington) 126, 400 (1957). 42. Dirscherr, W., H. Thomas und H. Schriefers, Acta endocr., K'hvn. 39, 385 (1962). - 43. Goodalt, McC., N. Krrshner und L. Rosen, J. Clin. Invest. 38, 707 (1959). - 44. KIRShNer, N., McC. Goodall und L. Rosen, Proc. Soc. exp. Biol. Med. 98, 627 (1958). - 45. La Brosse, E. H., J. Axelrod und S. S. Kety, Science (Washington) 128, 593 (1958). - 46. Rosen, I. und C. McGoodall, Proc. Soc. exp. Biol. Med. 110, 767 (1962). - 47. Symposion on Catecholamines, Bethesda /Md. 1958, Pharmacol. Rev. 11, 233 (1959). - 48. Udenfriend, S., E. Tirus und H. Weissbach, J. biol. Chemistry 216, 499 (1955). - 49. SANdLeR, M., in: The clinical chemistry of monoamines. Elsevier Publ. Co., Amsterdam (1963). - 50. Chadwick, B. T. und J. H. Wilkinson, Clin. chim. Acta (Amsterdam) 5, 879 (1960). - 51. Honet, J. C., T. V. Casey und J. W. Runyan, N. England J. Med. 261, 188 (1959). - 52. Mustala, O., J. J. Tuomisto und M. M. AirakSINEN, Scand. J. Clin. Lab. Invest. 16, 655 (1964). - 53. SAto, T., K. Yoshinaga, N. Ishida, C. ItoH und Y. WADA, Tohoku J. exp. Med. 77, 78 (1962). - 54. Hartmanin, F., Gatsroenterologia, Basel, Suppl. zu 95, 182 (1961). - 55. HARTMANN, F. und W. Ruge, Dtsch. Arch. klin. Med. 208, 298 (1962). - 56. Schmrd, E., L. Zrcha und W. JoHN, Zschr. Gastroenterol. 2, 214 (1964). - 57. v. Studnitz, W., K. Engelman und A. Sjoerdsma, Clin. chim. Acta (Amsterdam) 9, 224 (1964). - 58. Scrmmo, E., H. HAAs, N. Henning, K. MeythaleR jr. und H. SchöN, Klin. Wschr. 40, 1229 (1962). - 59. GJessing, L. R., Scand. J. Clin. Lab. Invest. 16, 661 (1964); Lancet $I I, 1281$ (1963). - 60. K̈̈̈SER, H., M. BETTEX und W. v. Studnitz, Atch. Dis. Childh., London 39, 168 (1964). 61. v. Studnitz, W., H. KäSER und A. Sjoerdsma, N. England J. Med. 269, 232 (1963). - 62. WiLliams, C. M. und M. Greer, Amer. J. Med. Ass. 183, 836 (1963). - 63. STURM, jr., A., Dtsch. med. Wschr. 88, 1000 (1963). - 64. Robinson, R., P. SMITH und S. R. F. WhITTACKER, Brit. Med. J. I, 1422 (1964). - 65. KARLSON, P., C. E. SEKERIS und P. HerrLICH, Dtsch. med. Wschr. 88,1873 (1963). - 66. v. Studnitz, W., Scand. J. Clin. Laborat. Invest. 11, 309 (1959). - 67. Carlsson, A., N.-A. Hillakp und B. Waldeck, Acta physiol. scand. 59, 215 (1963). - 68. Phirmppu, A., D. Palm und H. J. SchüManN, Nature (London) 205, 183 (1965). - 69. Schöne, H. H. und E. Lindner, Arzneimittel-Forsch. 10, 583 (1960); Klin. Wschr. 40, 1196 (1962). - 70. KrRBERGER, E. und U. Moнs, Med. Klin. 56, 2211 (1961). - 71. Kuschre, H. J., H. IDRISS und F. ECKMANN, Klin. Wschr. 43, 617 (1965). - 72. Rauen, H. M. und J. Aebert, Klin. Wschr. 42, 443 (1964). - $7 \dot{3}$. Stotr, A. W. und R. Robrnson, J. Pharmacy Pharmacol. 15, 773 (1963).
Priv.-Doz. Dr. E. Schmid I. Med. Klinik der Med. Akademie 24 Lübeck Kronsforder Allee 